\title{
INCIDENCES OF RESPIRATORY DISTRESS SYNDROME IN PRETERM INFANTS WHO WERE BORN WITHIN 24 HOURS OF DEXAMETHASONE BEING ADMINISTERED TO THE MOTHER VERSUS THOSE MOTHERS WITHOUT
}

\author{
M. Kashanian ${ }^{1}$, M. Morravegi Asl ${ }^{2}$, A. Ahangari ${ }^{2}$ \\ ${ }^{1}$ Obstetrics \& Gynecology, ${ }^{2}$ Tehran University of Medical Sciences, Tehran, Iran
}

Objective: A Comparison between the incidences of respiratory distress syndrome in preterm infants who were born within 24 hours of dexamethasone being first administered to the mother versus those mothers without.

Methods: A prospective study was performed on pregnant women with the complaint of preterm labor during 30-36 weeks of pregnancy and a probability of delivery within 24 hours. In the dexamethasone group (100 cases), drug was administered every. However, patients delivered within 24 hours of the first dexamethasone administration and before finishing the course of treatment. The control group (70 patients), did not receive dexamethasone, and delivered within 24 hours of admission .

Results: In the dexamethasone group the RDS were observed in $15 \%$ of neonates versus $27.1 \%$ in the control group $(\mathrm{P}<0.05)$. Between 30-31.6 weeks of pregnancy, the rate of RDS in the neonates of the dexamethasone group were $18.9 \%$ versus $35 \%$ in the control group $(\mathrm{P}=0.001)$. Between 32-33.6 weeks of pregnancy, the RDS were observed in $14.3 \%$ and $30.7 \%$ of neonates of the dexamethasone and control group respectively $(\mathrm{P}=0.001)$. Also between 34-36 weeks of pregnancy there were $10.7 \%$ RDS in the dexamethasone group versus $16.7 \%$ in the control group $(\mathrm{P}=0.004)$, which for all gestational ages have significant differences. RDS in male sex was more prevalent than female $(\mathrm{P}=0.001)$, and neonates who were born by cesarean section suffered from RDS more than vaginally born neonates $(\mathrm{P}=0.005)$.

Conclusion: It is suggested to administer corticosteroids for the patients whose delivery is eminent. 